posė à l'usine Bergès, à Lancey, pendant les essais de retour à la terre.

te 'Tenir la vitesse normale et la noter soigneusement.

2o Arriver, dans l'espace d'une minule environ, à la tension fixée, tenir celte tension bien constante et noter soigneusement.

$3^{\circ}$ Chaque jour, mettre en marche à midi précıs, marcher comme dit cl-rlessus jusqu'à midı quatorze, arrêter dans l'espace d'une minute, et laısser arrêter jusqu'à mirli trente. Puis remettre en marche dans les mêmes conditions jusqu'à midı quarantequatre, puis arrèter dans les mêmes conditions jusqu'à une heure, remeltre en marche jusqu'à une heure quinze, arrêter jusqu'à une heure trente, remettre en marche jusqu'à une heure cinquanle el arrèter ensuile définitivement.

L'application de ce programme s'effeclua de point en point. Chaque mise en marche et chaque arrêt se firent progresslvement, mais de façon que la manœuvre totale ne dura pas plus d'une minute.

Il convient enfin de remarquer que, le 31 août 1906, l'usine de Lancey a supprımé, pour metlre en évidence les influences dues au tramway de Grenoble à Chaparelllan el les autres, une pérıde de $1 / 4$ d'heure de marche $(1 \mathrm{~h}$. à 1 h. $1 / 4)$.

lésullats obtenus dans les essais à courants allernatifs.Les plus inléressants de ces essals sont évidemment constitués par la classe relative aux lignes, mises à la terre aux deux bouts, soumises à des effels inducteurs alternalifs. Nous donnons $\mathrm{cl}_{1}$ dessous le tableau résumé des résultats obtenus.

Résumé des Résultats.- Lignes à la terre aux deux bouts.Sous le bénéfices des hypothèses fartes, en représentant par $l$ la longueur en kilomètres de la ligne, par $S$ le produst $M \Omega 10^{-3}$, c'esl-à-dire, d'après ce que nous avons dit, la tension induite en millıvolts par ampère inducteur, nous pouvons définir un coefficlent $\sigma=\frac{S 10.3}{l \mathrm{~km}}$, comme représentant la tension induite en volts par kilomèlre de ligne et par ampère inducteur.

Les diverses expériences effectuées sur les diverses lignes indultes, mises à la terre aux deux extrémités, peuvent donc être résumées dans le tableau suivant:

$$
\begin{aligned}
& \text { Thésignation } \begin{array}{c}
\text { Longueur } \\
\text { indute mostance } \\
\text { monne de }
\end{array} \\
& \text { de la ligne } \\
& \text { endute moyenne de la } \\
& \text { a lia ligne intuite. }
\end{aligned}
$$

$$
\begin{gathered}
\text { Volts induits } \\
\text { par } \\
\text { ampere } \\
\text { in ructeur }
\end{gathered}
$$

Vo'ls induts

par anpère inducteur et par hilometrex
de ligne nutuctrire.

Le Versoud...... ; $2,500 \quad 8 \mathrm{~m} 25$

$$
563 \times 10^{3}
$$

$0,5 \pi 3$

Lancey. . . .

Theys.......

Craponoz

$7,600 \quad 4 \% \tau^{m} 70$

$$
3.0 \times 10^{-3}
$$

$0,3^{\prime} 0$ $=0,22520$

Montileury. .

$1.4,500 \quad 4.857 \mathrm{~m}$

$\frac{0,1757}{29}=00026 \frac{0,0026}{14,50}$

Quant aux eflets sur lignes béphoniques doubles, ll y a lieu de distınguer deux cas, celuı où les lignes n'élaient pas pourvues de croisement, et celul où elles en élaient pourvues.

Eu égard à la fablesse des eflets à mequrer, ces derniers résultals d'expérıences ont été falts simplement sur la lıgne LanceyVersoud (groupe $a$ ).

Sur la ligne sans croisements, les effels induits ont été de 0,167 volt (courant inducteur 30 ampères allernalifs, distance moyeune des lignes, 7 à 10 mètres. longueur du paralléliome, 2500 kloinètres). Quant aux mèmes lignes doubles dépourvues de croisements, isolées du groupe $a$, on n'a oblenu aucune déviation sensible de l'electrorynamomètre, et un contróle de la faiblesse de ces effets a été effectué par embrochage, sul ces lıgnes, d'un lélephone quı n'a donné nassance qu'à un son extrèmement fable.

Résullats obtenus dans les essais à courants continus.Ils devalent, iaturellement, donner lieu à une intensilé d'effels beaucoup moins considerables que ceux a courants viternalifs, la partie variable ou ondulée du courant pseudo-continu de la dynaino employée oscillant entre 1 et 2 milliampères pour 26 ampères inducteurs moyens. Les effets induils produits 'ont donc élé insıgnifiants, impercepibles même. Il est à peine utıle de dire que nous n'avons opéré, dans cet ordre de recherches, que sur les lignes du groupe $a$. Cependant, il ne convient pas de généralise. d'une manıère absolue la portée de ces résultats négatifs obtenus avec les courants continus. Il convient de remarquer que nous avons opéré sur une machine a courant continu de traction, donc de vollage moyen, et pour laquelle le rapport du nombre des lames du collecteur à la tension produite était réellement élevé.

Rien ne nous permet d'espérer que les effets du courant continu seralent tout à fait aussi inoffensifs avec une machine à couranl continu de tension élevée, dans laquelle le rapport du nombre des lames à la tension serait beaucoup plus pelıt.

Telles sont, brièvement résumées, les opérations expérimen. tales auxquelles nous nous sommes livrés durant les deux cam. pagnes d'été 1906 et 1907. A nous, simple expérimentateur chargé de la réalısation pratique d'une méthode d'essai, n'appartient pas pas de discuter les résultats obtenus el de mettre en lumière leup sujet. Le rapport d'ensemble présenté au comité d'électrıcilé par M. Harlé, pressdent de la commission, et qui contientin extenso, outre le comple-rendu de nos expériences, une magistrale étude de notre collègue et ami M. Brylinski, a sur l'interprétation des résullats oblenus et les conclusions qu'ils entraînent" jelle un jour définitif sur la question, et conshtue un document de haule valeur qui fera jurisprudence en la matière. Pour nous, dont le rôle est plus modeste, nous aurons été trop heureux si nous avons pu, au prix de nos efforts, réussir à rassembler quelques résultats pratiques de quelque utilité pour la si intéressante in. dustrie des transporls d'énergıe.

\section{LE LABORATOIRE D'ESSAIS DU CONSERVATOIRE NATIONAL DES ARTS ET MÉTIEPB}

(Suite.)

\section{II ${ }^{\circ}-$ GROUPE DES ESSAIS HYDRAULIQUES $\left(^{*}\right)$}

Ensemble de l'installation. - Le groupe des essais hydrauliques est installé dans un local parallèle à la rue Vaucanson, et dit a sous-sol Vaucanson ». La plancheI montre la disposition essentielle de la station des essais hydrauliques. Le sol est en béton. dans l'épaisseur duquel sont noyés des fers à double $\mathrm{T}$ assemblés parallèlement et solidement scellés, qui permettent le montage des machines à soumettre aux essais, en un point quelconque de la salle.

Dans l'axe de cette salle sont creusés deux canaux $\mathrm{SP}_{1} \mathrm{I}$ et $S \mathrm{P}_{2} \mathrm{I}$, terminés en $\mathrm{S}$ chacun par un déversoir. Ces canaux font communiquer la citerne $A$, d'une capacité de 20 mètres cubes environ, avec une citerne $\mathrm{I}$, d'une profondeur de $2 \mathrm{~m} 50$, qui sert de chambre de repos, et permet également l'instal. lation des turbines devant fonctionner noyées.

Une pompe $\mathrm{C}$ vient s'almenter dans la citerne $\mathrm{A}$; elle refoule son eau dans la conduite $\mathrm{E}$ d'où elle alimente les turbines en essai $T$; l'eau est déversée par la turbine dans la chambre $I$, d'où elle fait retour à la citerne $A$, après avoir traversé les déversoirs $S$ dont l'utilité est de mesurer l'eau débitée par les appareils en essais. Du reste, nous revien. drons plus loin sur la description de nos déversoirs. Au plafond, ainsi que sur le sol de la salle d'essai, sont fixées un certain nombre de conduites de dimensions différentes $B, D, E, V$ (etc.), que l'on peut voir sur la planche I, et qui servent à l'organisation des divers essais.

La pompe ( constitue pour le Laboratoire une véritable petite chute de montagne; elle peut débiter i 35 litres par seconde sous 58 mètres de charge. Cette pompe est actionnée par deux moteurs électriques $\mathrm{M}_{1}(100 \mathrm{HP})$ et $\mathrm{M}_{2}$ (50 HP), pouvant ensemble développer i 50 à 200 chevaux.

(*) Avant d'aborder la description du groupe des essais hydrauliques, nous tenons à rappeler ici l'appui qu'a bien voulu nous accorder la Chambre Synticale des Forces Hydrauliques qui, dès igo3, avait nommé une commission d'etude des essais hydrauliques (Voir La Houlle Blanche d'octobre 1903 ).

Cette commission avait élaboré, en ce qui concerne les essais turbunes que nous allons décrire plus loin, un programme de travaux dont elle a confie la réalısation à notre Laboratorre, en mettant à sa disposition les moyens d'execulion. Nous sommes heureux de rendre hommage au coucours precieux que nous ont apporté, en toutes circonstances, son regretté president, M. Pinat, son president actuel, M. Cordien, et tous ses membres. 

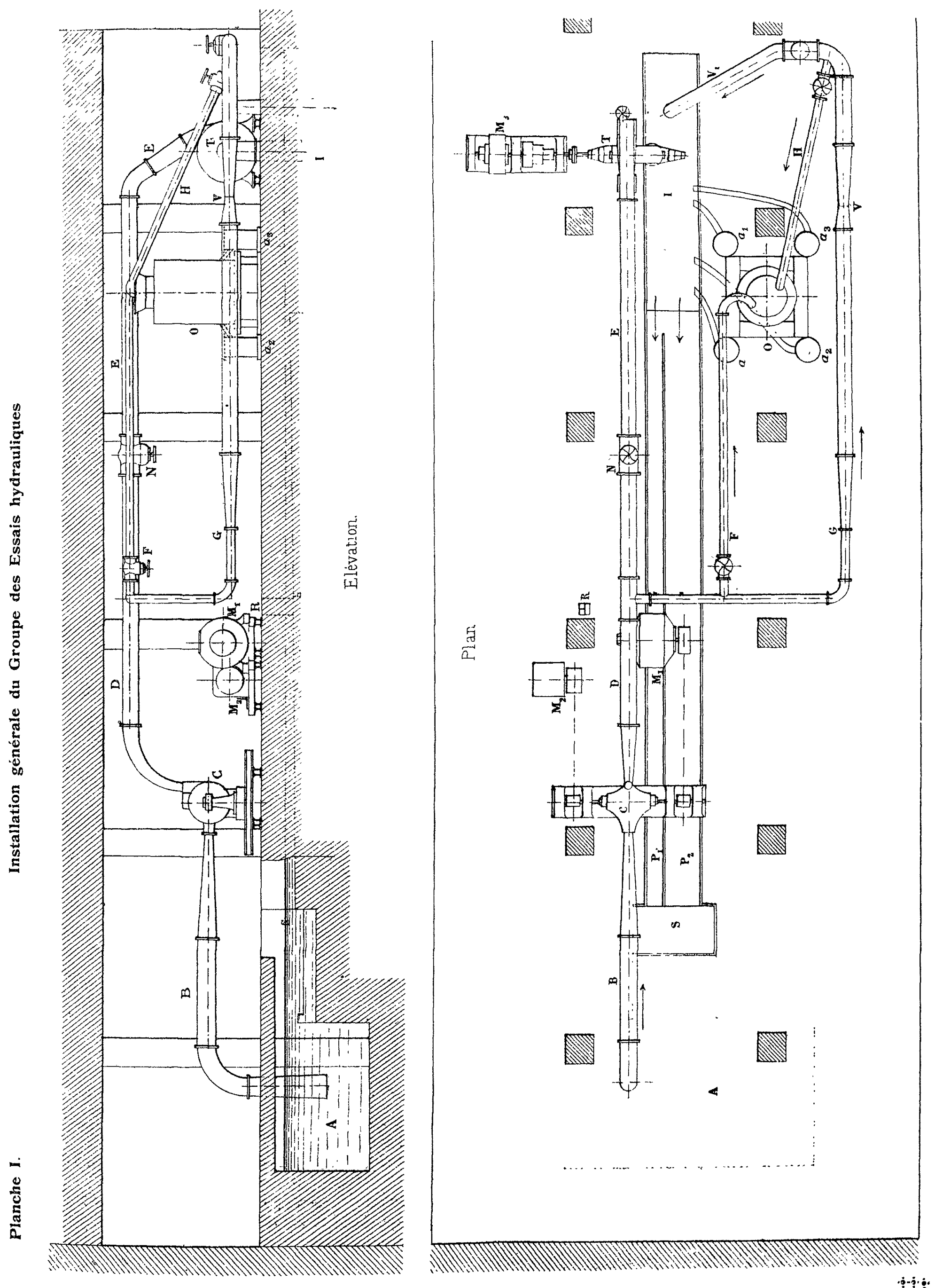
A la pompe C, l'on peut substituer une autre pompe donnant 500 litres par seconde sous 2 ou 3 mètres de charge seulement, elle est destinée à l'exécution des essais qui demandent un plus grand débit et une charge moindre.

Comme on le voit, il est facile, en utilisant la pompe voulue, de créer pour chaque essai la chute nécessaire; c'est la pompe $\mathrm{C}$ qui est le plus généralement employée. Nous en donnerons plus loin une description détaillée, qui nous fournira l'occasion de donner un exemple de la maniere dont nous exécutons un essai de pompe; mais nous voulons tout d'abord fixer les détails des instruments de mesure employés dans nos essais hydrauliques.

Des appareils de mesure employés dan ́ les essais hydrauliques. - Pressions, - Les pressions, ou hauteurs de chutes d'eau dans le cas des turbines, et hauteurs de refoulements dans le cas de pompes, sont mesurées à l'aide de manomètres métalliques pour les très hautes pressions, de manomètres à mercure pour les moyennes pressions, et enfin de manomètres à eau pour les très faibles pressions. Le mercure, ou l'eau, sont renfermés dans des tubes en

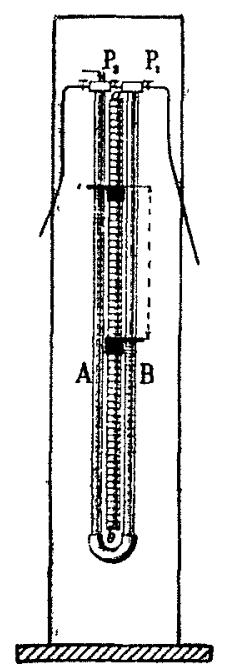

Fig. 4 .

Manomètre. verre en forme d'U; parallèlement à ces tubes, une graduation $a b$, munie de curseurs, permet de lire avec précision la hauteur de charge. Des robinets à plusieurs voies, $\mathrm{P}_{1}$ et $P_{2}$, sont convenablement disposés pour pouvoir purger d'air les parties hautes des conduites, ou les manomètres eux-mêmes. Ces manomètres sont représentés par la figure 4 , sur laquelle $A$ et $B$ sont des tubes de verre ; $a b$ est une règle graduée en millimètres. Le robinet $\mathrm{P}_{1}$ est relié à la poinpe dans le cas de l'emploi du mercure, ou à un réservoir d'air lorsqu'on emploie de l'eau.

Puissances. - La mesure de la puissance dépensée dans le cas des pompes, et de la puissance recueillie dans le cas des turbines ou des moteurs hydrauliques, se fait à l'aide d'appareils de mesure électriques de haute précision, étalonnés avant et apès chaque essai. On tient compte, bien entendu, du rendement des machines et des transmissions, s'il y a lieu. Dans certains cas, on a recours aux freins de Prony ou aux dynamo-dynamométriques. Ces derniers seront décrits plus loin dans le chapitre qui traitera des essais d'hélices aériennes, et des moteurs thermiques.

Débits. - Connaissant la pression et la puissance consommée, ou recueillie, il ne nous reste plus qu'à jauger avec précision l'eau débitée pour avoir en main le troisième élément d'un essai hydraulique complet. Les jugeages d'eau se font avec trois appareils différents, survant l'importance des débits à mesurer.

S'il s'agit de petits débits, compris entre o et 100 litres par minute, on utilise des hectolitres gradués, et vérifiés par peséc. Pour les débits moyens, de 1,5 a r o litres par seconde, on opère avec une bàchè à orifices d'écoulement, et pour les débits supérieurs à 40 mètres cubes à l'heure, on utilise deux déversoirs dont la capacité peut dépasser I 000 mètres cubes à l'heure.

Hectolitres. - Les hectolitres que possède le Laboratoire sont cylindriques, et gradués de litre en litre, depuis 50 litres jusqu'à r 10 litres. La graduation occupe l'axe du cylindre, pour éviter sur la lecture l'intluence d'un défaut d'horizontalité.

Bâche à orifices. - La bâche à orifices (fig. 5) est cons- tituée par un grand cylindre B, de $\mathrm{I}^{\mathrm{m}} 20$ de diamètre, servant de réservoir d'eau, dans l'axe duquel est introduit un cylindre plus petit $\mathrm{A}$, de om 60 de diamètre, terminé en haut par un entonnoir grillagé pour l'arrivée de l'eau; ce cylindre est sans fond, il est fixé au grand par des entre. toises P. On a employé pour leur construction des tôles galvanisées de $2 \mathrm{~mm}$. d'épaisseur; le fond du grand cylindre est en tôle de $3 \mathrm{~mm}$. Quatre amorces de conduites I, 2,3 et 4 , sont fermées par un fond plat, en tôle de $10 \mathrm{~mm}$. d'épaisseur, portant 4 orifices en bronze dur en mince paroi, de différents diamètres. La figure 6 donne, à plus grande échelle, la coupe de l'un de ces orifices.

Un robinet de vidange $\mathrm{R}$ permet de vider complètement la bâche. Le robinet $\mathrm{N}$ fait communiquer l'intérieur de la bâche avec un tube en verre porté par une graduation décimale remontant jusqu'à sa partie supérieure. La hauten? totale de la bâche est de $\mathrm{i}$ m 50 .

Cette bâche est installée en $O$ de la planche I, sur un socle en madriers de bois, parfaitement horizontal Le dimensions de l'appareil sont telles que le comportent les diamètre des orifices d'écoulement. Dans ces conditions, on

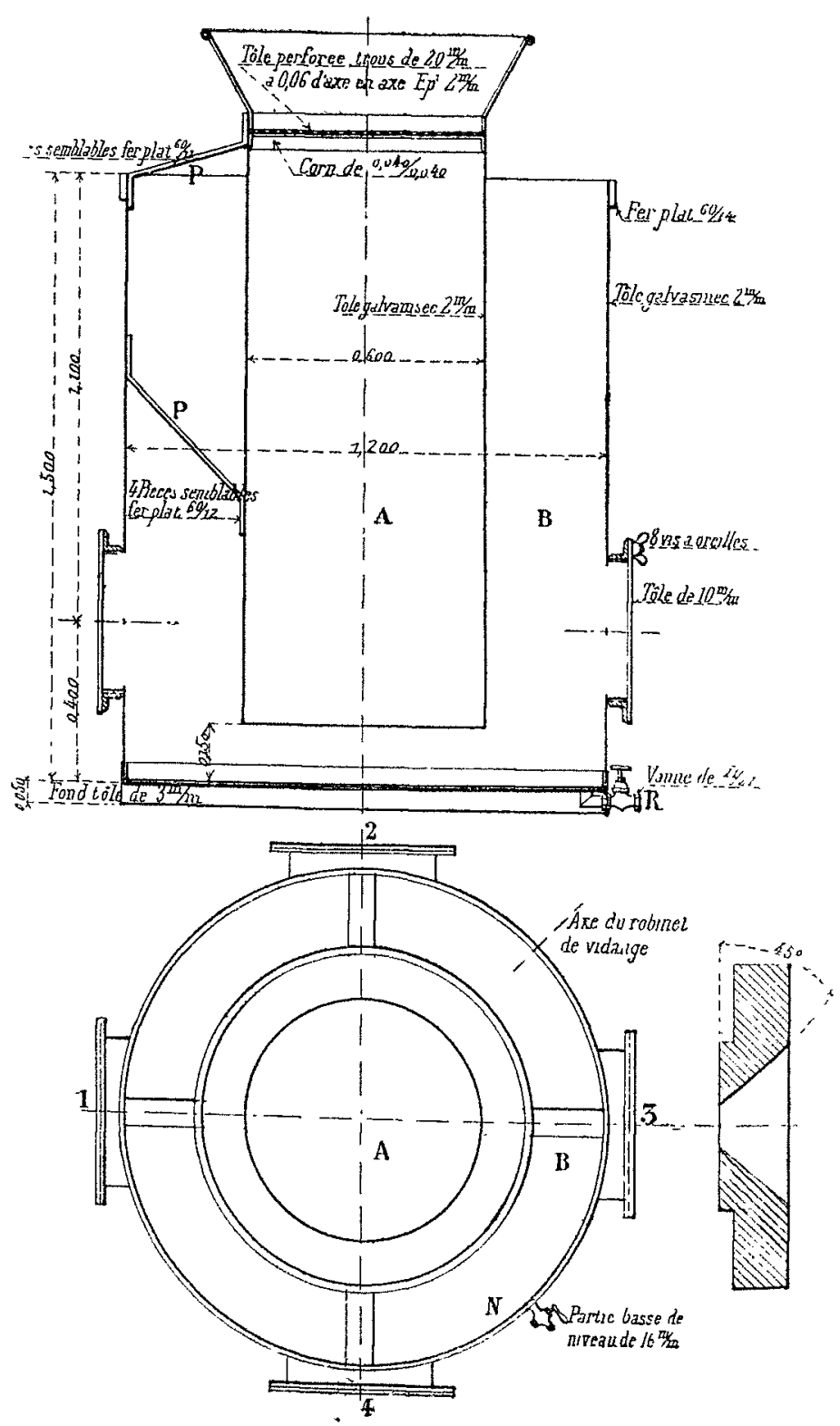

FiG. 5 et 6. - Détalls de la bâche à orifices.

aurait pu admettre pour cet appareil le coefficient de débit o,joo, donné par la plupart des auteurs; mais, dans le cas où les 4 orifices fonctionnent ensemble, l'eau prend dans, la bâche un mouvement de rotation qui trouble légèrement la 
netteté des jets; dans ces conditions, il devenait indispensable de jauger expérimentalement les débits des orifices, sous la charge normale de un mètre adoptée.

La bàche fonctionnant avec un $1,2.3$ et 4 orifices ouverts à la fois, l'eau a été recueillie dans des bacs pesés. On a ansi vérifié, par pesée de l'eau, les coefficients de débits correspondants. Les résultats de ces expériences sont condensés dans le tableau suivant:

Résultats du tarage de la bâche à orifices

\begin{tabular}{|c|c|c|}
\hline $\begin{array}{c}\text { Diamètre } \\
\text { des orifices }\end{array}$ & $\begin{array}{c}\text { Coeffients } \\
\text { de débit }\end{array}$ & $\begin{array}{l}\text { Hauteur de charge } \\
\text { sous laquelle } \\
\text { fonctionnat la bàche }\end{array}$ \\
\hline \multicolumn{3}{|c|}{ UN SEUL ORIFICE OUVERT : } \\
\hline $\begin{array}{l}40 \mathrm{~m} / \mathrm{m}^{\mathrm{m}} \\
30 \mathrm{~m} / \mathrm{m}\end{array}$ & $\begin{array}{l}06000 \\
0,6080\end{array}$ & $\begin{array}{l}\mid m j 39 \\
1^{m 041}\end{array}$ \\
\hline \multicolumn{3}{|c|}{2 ORIFICES $(1+T 2)$ OUVERTS SIMULTANEMEN } \\
\hline $\begin{array}{l}10 \mathrm{~m} / \mathrm{m} \\
30 \mathrm{~m} / \mathrm{m}\end{array}$ & $\begin{array}{l}0,6012 \\
0,6078\end{array}$ & $\begin{array}{l}\operatorname{lm} 010 \\
1 \mathrm{~m} 0 \mathrm{~L} 0\end{array}$ \\
\hline \multicolumn{3}{|c|}{3 ORIFICES (1, 2 ET 4 ) OUVERTS SHULTAN ÉMENT } \\
\hline $\begin{array}{l}40 \mathrm{~m} / \mathrm{m} \\
30 \mathrm{~m} / \mathrm{m} \\
30 \mathrm{~m} / \mathrm{m}\end{array}$ & $\begin{array}{l}0,6007 \\
0,6084 \\
0,6103\end{array}$ & $\begin{array}{l}1 \mathrm{mr} 49 \\
1 \mathrm{w} 043 \\
1 \mathrm{~m} 039\end{array}$ \\
\hline \multicolumn{3}{|c|}{4 ORIFICES OUVERTS SIMULT INÉMLNT } \\
\hline $\begin{array}{l}40 \mathrm{~m} / \mathrm{m} \\
3 / \mathrm{m} / \mathrm{m} \\
40 \mathrm{~m} / \mathrm{m} \\
31 \mathrm{~m} / \mathrm{m}\end{array}$ & $\begin{array}{l}0,60: 38 \\
0,6097 \\
0,6029 \\
0,6148\end{array}$ & $\begin{array}{l}1 \mathrm{~m} 044 \\
1 \mathrm{~m} 013 \\
1 \mathrm{~m} 049 \\
1 \mathrm{~m} 038\end{array}$ \\
\hline
\end{tabular}

Déversoirs. - Enfin, our les gros débits, on utilise les déversoirs dans l'exécution desquels nous nous sommes inspirés des très belles études de $M$. Bazin sur la matière $\left(^{*}\right)$. Malheureusement, les dimensions du local n'ont pas permis de reproduire exactement le déversoir-type de $M$. Bazin, de telle sorte que, comme dans le cas de la bâche, il est devenu indispensable de déterminer expérimentalement les coelficients de débits de notre appareıl.

Ces très longues expériences, dont nous ne donnerons ici qu'un très succint résumé, sont publiẻes en détall dans le no 5 du Bulletin du Laboratoire des Arts et Métiers. Le Laboratoire possède maintenant un appareil permettant d'évaluer le débit d'une machine hydraulique à moins de I pour 100 près. La planche I montre les deux rigoles $P_{1}$ et $P_{2}$ des déversoirs, terminées chacune par un déversoir en mince paroi sans contraction latérale, et disposé de manière à permettre à l'air emprisonné sous la nappe de s'écouler convenablement sans laisser de pression sous elle. Chacune des rigoles se trouve communiquer avec les puits $R$ (voir Pl. II) par une conduite souterraine; dans ces puits plongent deux règles à pointes renversées, permettant de mesurer avec une très grande précision le niveau de l'eau et, partant, la charge sur la crête des déversoirs. Nons décrirons tout d'abord cet appareil accessoire.

Appareil pour mesurer les hauteurs de charge des déversoirs. - La planche II représente les détails de la règle à pointe renversée dont l'idée, très originale, est due à M.M. Bazin et Hégly; c'est celle qu'lls ont utilisée dans leurs expériences de déversoirs déjà citées plus haut.

( $\left.{ }^{+}\right)$Bazin. Annales des Ponts-et-Chaussées, 1888 (octobie).
Le puits $\mathrm{R}$ est situé à 5 mètres en amont du déversoir et est divisé en trois compartiments $A, B$ et $C$. A et $B$ sont respectivement en communication avec les rigoles $P_{1}$ et $P_{g}$, (Pl.l)par une conduite souterraine venant aboutir aux orifices $O$ et $\mathrm{O}^{\prime}$. Par les orifices $s$ et $s_{1}$, qu'on peut obturer par des bouchons, on peut faire communiquer, soit le compartiment $A$, soit le compartiment $B$, avec le compartiment $C$. Ce dernier contient un flotteur $T$, dont la tige est en connexion avec un enregistreur à mouvement d'horlogerie, inscrivant sur une feuille spéciale la courbe des charges du déversoir avec lequel on opère.

Dans les compartiments $A$ et $B$, plongent les tiges $R_{1}$ et $R_{2}$, terminées à leurs extrémités inférieures par les pointes renversées. Leurs extrémités supérieures sont solidement assemblées, et se terminent par une tige unique en laiton qui peut glisser à frottement doux dans le manchon E. En produisant ce glissement à la main, on amène la pointe de la règle à peu près en contact avec le niveau de l'eau; on manœuvre alors le bouton fileté D qui, serrant un collier, vient rendre la tige à pointe solidaire du manchon E. Ce manchon fileté extérieurement porte un écrou molleté $F$ venant reposer sur le support $K$ de la règle. En tournant à la main l'écrou $F$, on fait monter ou descendre la tige a pointe, et l'on amène cette dernière à coïncider exactement avec la surface du liquide. Une buttée $H$ limite le mouvcment de bas en haut, celui de haut en bas étant limité par le contact du bouton E sur l'écrou F. La tige porte-pointe est solidaire d'un vernier $N$ qui se déplace sur une régle $M$ fixée sur les deux supports $K$ et $K$ ' qui servent en même temps de guidage aux tiges à pointes. Ces deux supports sont de fortes consoles en fonte, solidement boulonnées sur un pilier en maçonnerie.

La règle $M$ a été graduée avec grand soin, et vérifiée, sur les comparateurs du Laboratoire. Le point zéro, ou charge nulle des déversoirs, a été repéré par plusieurs expériences très soignées.

Le contact de la pointe renversée avec la surface du liquide se constate avec une précision extrême, en ayant soin d'observer la pointe, en la faisant se détacher sur l'image d'un écran blanc, vu par réflexion surla surface du liquide; dans ces conditions, dès que la pointe entre en contact avec la surface, elle produit un petit ménisque qui se décelle immédiatement par un petit point noir sur l'écran blanc.

Le flotteur, d'un genre un peu spécial par son guidage très soigné, porte une tige $T$ manœuvrant directement le crayon de l'enregistreur. Ce dispositif évite toute paresse dans l'inscription de la courbe, chose qui se présente souvent avec les connexions souples par fils d'acier. Ainsi conçu, cet enregistreur a donné toute satisfaction. Il n'est utilisé que pour avoir l'allure génćrale de l'expérience, les lectures des hauteurs de charge se faisant toujours directement sur le vernier de la règle. On fait, en général, pendant toute la durée d'un essai, une lecture par minute.

Tarage des déversoirs. - La planche I montre le dispositif expérimental que nous avons adopté dans le taráge de nos deux déversoirs.

La vanne $\mathrm{N}$ est fermée, une conduite dérivée $\mathrm{F}$ permet d'alimenter la bàche à orifices, placéc ea $O$, autour de laquelle 4 tonneaux $a, a_{1}, a_{2}, a_{3}$, convenablement disposés, recueillent l'eau des 4 orifices et la déversent à l'aide de manches en toile dans la chambre $I$, en tête des rigoles des déversoirs. Une conduite $G$ permet d'alimenter un compteur Venturi $V$ dont l'eau fait retour dans la chambre 1 par 
la conduite $V_{1}$; enfin, une dérivation $H$ permet d'alimenter la bâche après le Venturi.

Io Petit déversoir. - Il est facile, d'après la description qui précède, de se rendre compte, en regardant la planche I, que, par un jeu convenable des vannes, on peut alimenter la bàche à orifices $\mathrm{O}$, soit directement par la canalisation $\mathrm{F}$, soit indirectement par les canalisations $G$ et $H$. Dans ces conditions, on peur à volonté réaliser les' 3 combinaisons suivantes:

1) On alimente la bâche et le compteur par $\mathrm{G}$ et $\mathrm{H}$;

2) On alimente le compteur seul par G.

3) On alimente le compteur par G, et la bâche par F.

La bâche, avec les 4 orifices ouverts, pouvait débiter environ 10 litres par seconde.

En utilisant la combinaison 1) bâche et compteur, on cherche le coefficient de débit du petit déversoir, pour 10 litres par seconde.

Pour trouver ce chiffre, ainsi que les suivants, nous procédons par groupes de 4 expériences, qui compren-
Dans les expériences précédentes, le petit déversoir fonctionnait seul, le grand étant fermé par une cloison placée près de sa crête. Cette cloison était rendue soigneusement étanche au moyen d'un tuyau en caoutchouc, disposé sur 3 de ses côtćs, et gonflé d'air après la mise en place de la cloison.

2) Grand déversoir. - Le petit déversoir, ainsi taré jus. qu'à go litres par seconde, permet de tarer le grand jusqu'à ce même débit. Pour les débits supérieurs, on procèdera comme plus haut, le petit déversoir jouant cette fois le rôle attribué précédemment à la bâche.

On opère alors par les combinaisons suivantes:

4) On alimente le compteur et le petit déversoir seul;

5) On alimente le compteur-et le grand déversoir-seul;

6) On alimente le compteur et les deux déversoirs simultanément.

La combinaison 4) permet de tarer le compteur pour 90 litres, la combinaison 5) permet de tarer le grand_déversoir pour 90 litres également, puis, en conservant au grand

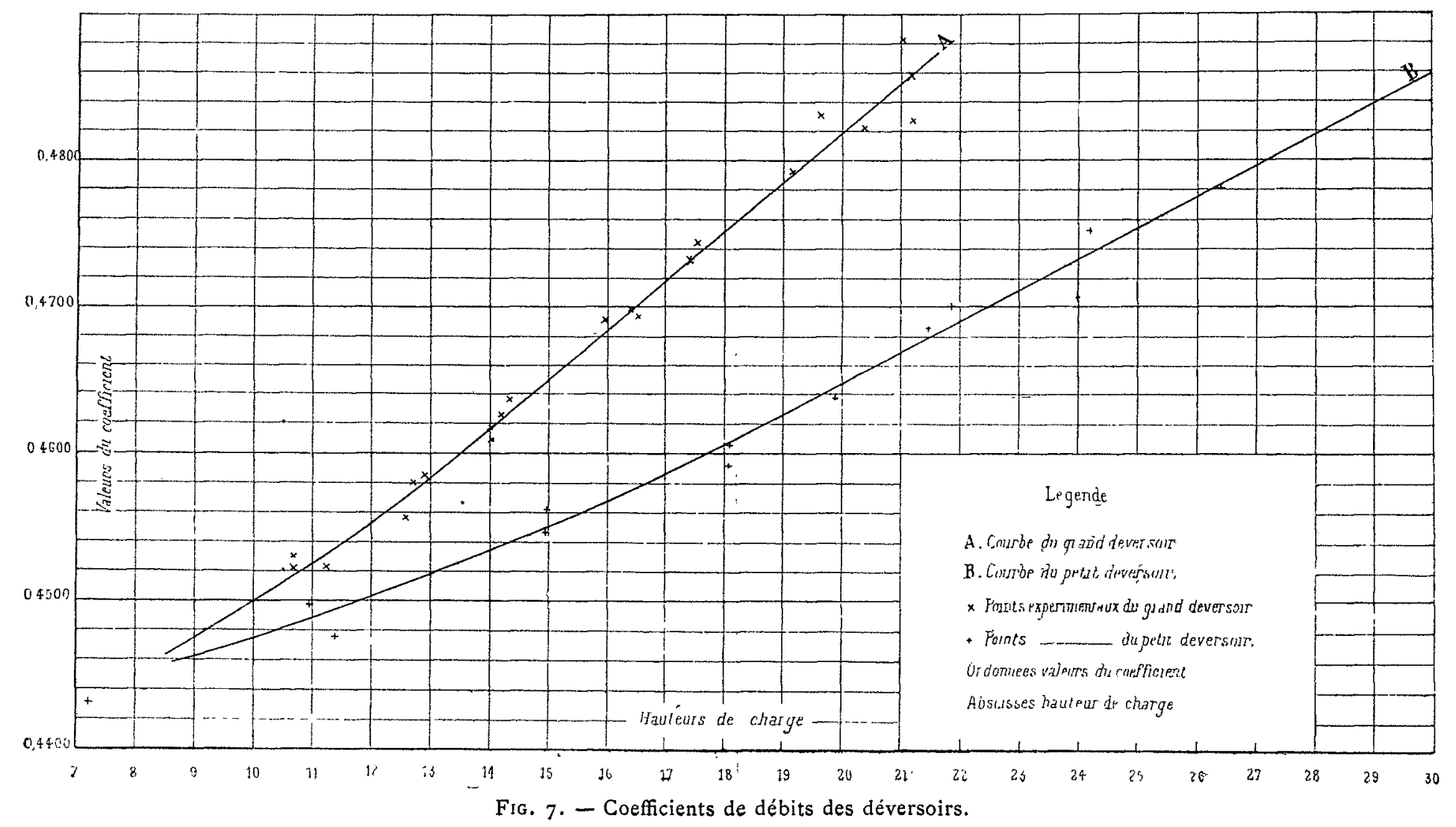

nent chacune environ io lectures de la règle donnant là hauteur de charge du déversoir, et io lectures de la règle donnant la hauteur de charge au dessus des orifices de la bàche. Ces lectures ont été faites à des intervalles de temps réguliers (une minute). Le coelficient adopté est la moyenne des 4 résultats obtenus (suffisamment concordants). Nous aurons ainsi du même coup taré le compteur pour ce débịt de ro litres par seconde.

Utilisant maintenant la combinaison 3), le compteur alimenté par $G$, et la bâche par $F$, on pourra tarer le déversoir pour 20 litres, puis, à l'aide du coefficient obtenu ainsi pour le déversoir, tarer, par la combinaison 2), le compteur seul par $G$, le compteur pour 20 litres, puis, par la combinaison 2), le déversoir pour 30 litres, et par la combinaison 3), le compteur pour 30 litres, etc... et ainsi de suite de proche en proche, jusqu'à la limite de débit du déservoir. On a ainsi atteint 90 litres par seconde environ, correspondant pour le petit déversoir à une hauteur de charge de om²g. déversoir ce débit de go litres, la combinaison 6) permettra de tarer le compteur pour un débit supérieur, et ainsi de proche en proche.

D'ailleurs, on a répété ces mesures en partant de débits plus faibles pour le petit déversoir, et l'on a obtenu une série de coefficients d'une façon indépendante les uns des autres, qui se sont groupés sur une même courbe .

On a procédé comme plus haut, chaque chiffre est la moyenne de 4 séries de groupes de ro lectures au moins.

D'aprèsl'étude de la méthode de mesure, on peut affirmer que ces déversoirs nous permettent de mesurer un débit avec une précision supérieure à I pour $100\left(^{*}\right)$.

On trouvera ci-joint deux tableaux résumant les résultats obtenus dans ces tarages. Les courbes de la figure 7 sont les traductions graphique des chiffres de ces deux tableaux. Les points expérimentaux sont reportés sur les

$\left({ }^{*}\right)$ Voir à ce sujet le Bulletm $\mathrm{n}^{*} 15 \mathrm{du}$ Laboratorre d'Essai, pages 17 et 18 . 


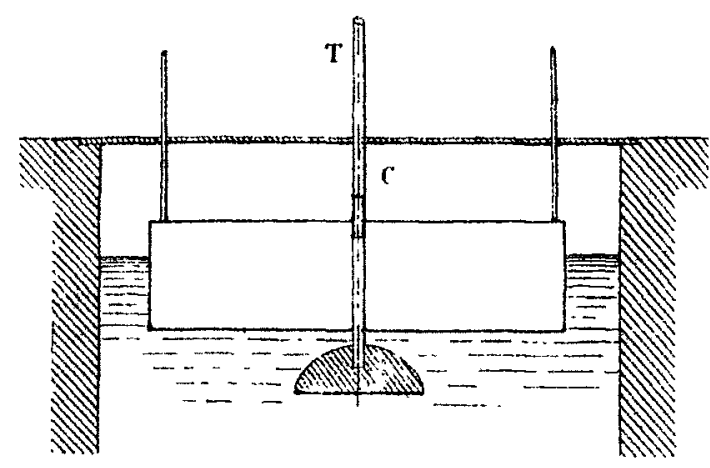

Fig 1 . Coupe verticale. $\mathbf{f g}$.

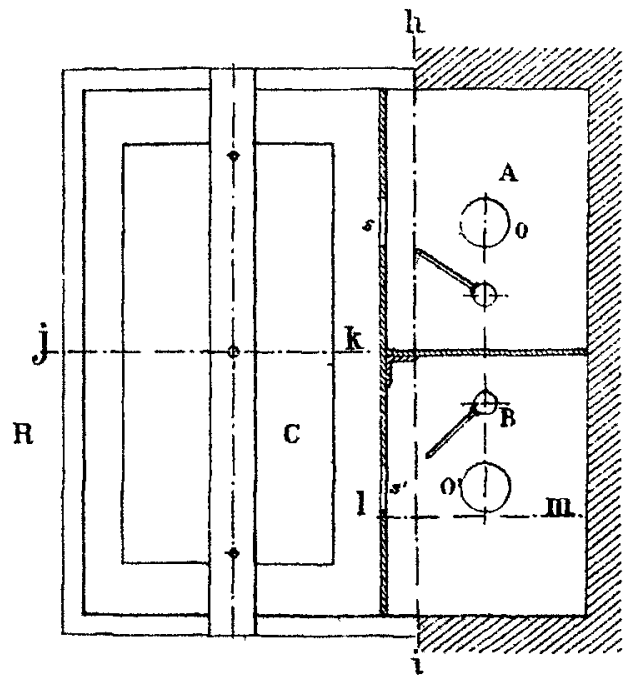

Fig 2 Coupe horizontale a b c de.

ig 3. Coupe verticale j klm

Frg.5. Coupe verticale hi.

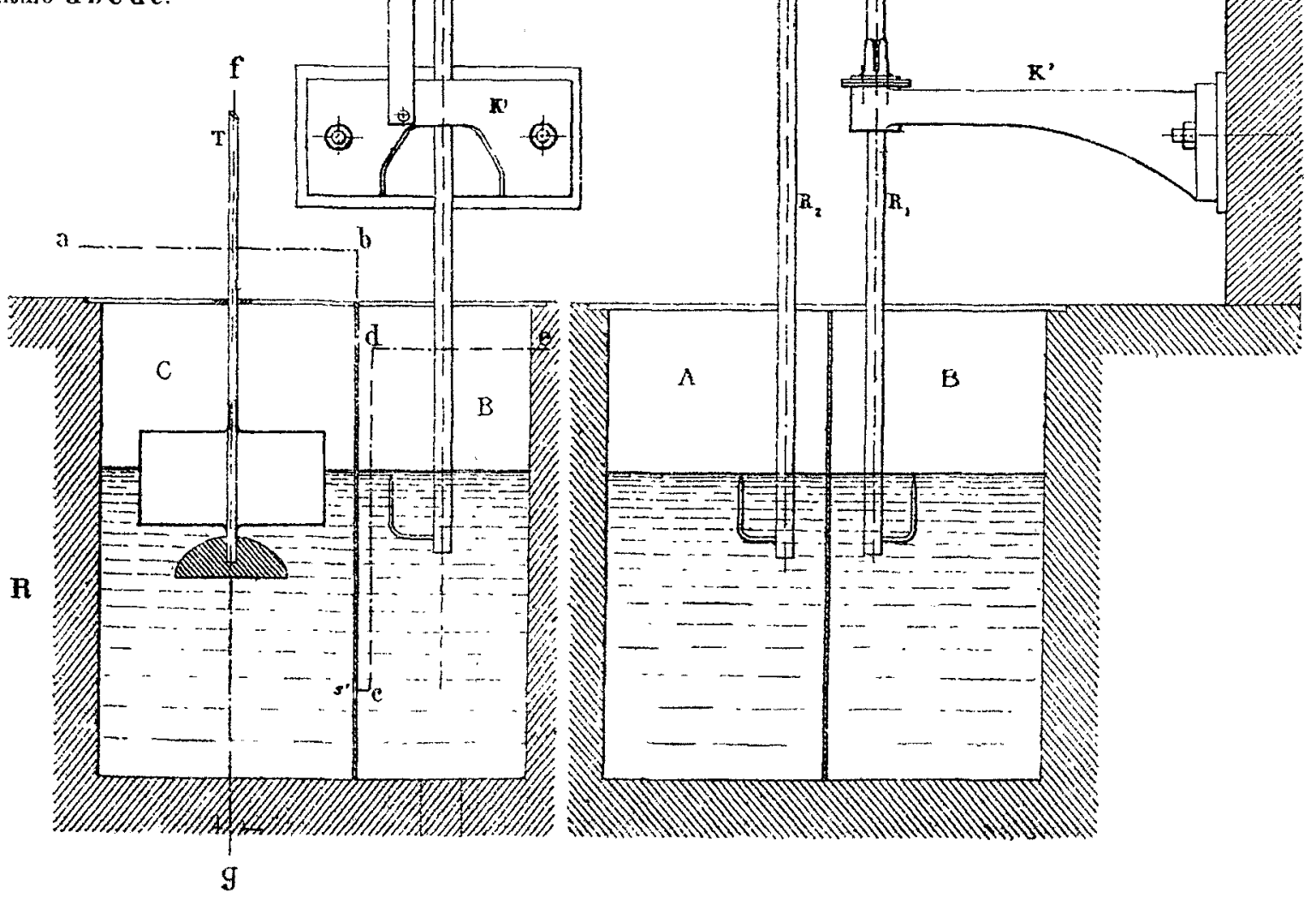


courbes pour donner au lecteur une idée de la précision et du nombre des mesures effectuées. Chaque point est la moyenne d'une série de i 2 ou 16 lectures.

Résultats du tarage des déversoirs

I. Tarage du petit déversoir (largəur $=0^{m_{2}} 80$ )

Volr figure $\overline{7}$

\begin{tabular}{|c|c|c|}
\hline Hauteur de charge & $\begin{array}{l}\text { Coefficient de débit } \\
\text { Résultats d'essais }\end{array}$ & $\begin{array}{l}\text { Coefficient de débit } \\
\text { lu sur la courbe }\end{array}$ \\
\hline $\begin{array}{ll}0 \mathrm{~m} 0721 \\
0 & 1094 \\
0 & 1138 \\
0 & 1491 \\
0 & 1497 \\
0 & 1811 \\
0 & 1813 \\
0 & 1991 \\
0 & 2147 \\
0 & 2188 \\
0 & 2401 \\
0 & 2193 \\
0 & 2(1.43 \\
0 & 9901 \\
0 & 2911\end{array}$ & $\begin{array}{l}0,4431 \\
0,4197 \\
0,4476 \\
0,4516 \\
0,4501 \\
0,4691 \\
0,1605 \\
0,4637 \\
0,1685 \\
0,4700 \\
0,4706 \\
0,4752 \\
0,4781 \\
0,4836 \\
0,4827\end{array}$ & $\begin{array}{l}0,4138 \\
0,1488 \\
0,4493 \\
0,4519 \\
0,4700 \\
0,4077 \\
0,46,37 \\
0,1615 \\
0,4678 \\
0,1637 \\
0,4732 \\
0,1736 \\
0,4733 \\
0,1838 \\
0,1810\end{array}$ \\
\hline
\end{tabular}

$2^{\circ}$ Tarage du grand déversoir (largeur om652b)

Voir figure 7 .

\begin{tabular}{|c|c|c|}
\hline Hauteur de charge & $\begin{array}{l}\text { Coefficient de débit } \\
\text { Résullats d'essais }\end{array}$ & $\begin{array}{l}\text { Coefficient de debit } \\
\text { lu sur la courbe }\end{array}$ \\
\hline $\begin{array}{ll}0 \mathrm{~m} 1067 \\
0 & 1068 \\
0 & 1123 \\
0 & 1257 \\
0 & 1269 \\
0 & 1287 \\
0 & 1402 \\
0 & 1403 \\
0 & 1420 \\
0 & 1435 \\
0 & 1595 \\
0 & 1641 \\
0 & 1651 \\
0 & 1742 \\
0 & 1754 \\
0 & 1918 \\
0 & 1964 \\
0 & 2040 \\
0 & 2105 \\
0 & 2120 \\
0 & 2122\end{array}$ & $\begin{array}{l}0,1530 \\
0,4592 \\
0,4529 \\
0,1353 \\
0,4580 \\
0,4585 \\
0,1617 \\
0,4609 \\
0,4696 \\
0,4637 \\
0,4691 \\
0,4698 \\
0,1683 \\
0,4731 \\
0,4744 \\
0,4792 \\
0,4890 \\
0,4829 \\
0,4889 \\
0,1858 \\
0,4827\end{array}$ & $\begin{array}{l}0,4515 \\
0,4515 \\
0,4530 \\
0,4569 \\
0,1574 \\
0,4579 \\
0,4613 \\
0,4615 \\
0,4692 \\
0,4697 \\
0,4681 \\
0,4696 \\
0,1700 \\
0,4731 \\
0,4735 \\
0,4790 \\
0,1800 \\
0,4831 \\
0,4853 \\
0,4858 \\
0,4859\end{array}$ \\
\hline
\end{tabular}

L'installation générale déjà décrite permet l'essai de pompes et de machines hydrauliques importantes; les quelques exemples qui vont être donnés dans un prochain article montreront clairement l'application de ces appareils de mesures.

(A suivre).

Boyer-Guillon, Ingénieur cinil des Mines,

Chef de la section des essais de machines au Laboratoire d'Essats du Conservatore Natzonal des Arts et Métiers.

\section{COINSTRUTCTIONT}

DES

\section{LIGNES DE TRANSPORT D'ÉNERGIE}

Rapport présenté au Congrès de Marsellle par M Le Rov, directeur du reséa du Var à la Societe Energie electrique du Littoral mediterraneen

Le choix des supports d'une ligne de transport d'énergie dépend principalement de trois facteurs : Sécurité de l'explostalion, prix de premier établissement, coût de l'entretien.

Nous nous occuperons donc tout d'abord des accidents des lignes électriques à haute tension, en étudiant les moyens de diminuer la fréquence de ces accidents, et l'influence du choix du support.
1. Court-circuil provenant du contact de branches d'arbres. de chutes d'arbres, de chutes de nierres, du contact d'ani. maux, ou du contact direct des fils entre eux. - Il est facile de se prémunir contre les chutes d'arbres ou contact de branches en élaguant largernent et abattant les arbres silués dans le voisinage immédıat de la ligne. On dolt considérer comıne une règle absolue qu'aucune branche ne dolt être à une distance moindre de $5 \mathrm{~m}$. des fils de ligne.

Le tracé de la ligne devra être étudié de façon à éviter autant que possible les grandes traversées de bois, el de façon à évilep aussi de placer la ligno en contre-bas d'éboulıs ou de rocher's pell stables. Dans les régıons très montagneuses, cette dernière ind. calıon n'est pas très facile à suivre; les lignes à grandes porlées favoriseront alors la sécurité du transport en permettant de sauter de contrefort en contrefort.

Les animaux qui viennent le plus souvent en contact avec les fils sont les oiseaux, et quelque mammiferes grimpeurs. L'inter. ruption qu'lls occasionnent est, en général, momentanée. Dans les transports à haute tension, dont les fils sont très écartés, c* genre d'interruption est moins fréquent avec les supports peu conducteurs (bois, par exemple) qu'avec les supports mélallıques. Ces interruptions peuvenl. d'allleurs, ètre complètement évılées dans le cas de transport à fils très écartés, en ne meltanl pas li, centre de la distribution à la terre

Les contacts directs entre fils de ligne se produisent quelquefors lorsque plusieurs fils sont sur un même plan horizontal. Cetle dıspositıon est fréquemment adoptée en Amérique, où l'on admel que l'écartement entre tils doit être au moins égal à lib mollié de la flèche. Dans le cas de très grandes porlées $(300 \mathrm{~m}$. ou $400 \mathrm{~m}$ ), il est préférablé de placer les conducleurs à des niveaux différents.

2. Supporls détruits ou abattus. Les supporls d'une ligne peuvent brîler dans un incendie de foréts, être brîlés par le courant, $\hat{e}$ lre renversés par un vent violent, ou parce que les eaux ont affounllé leurs fondations. - Une large tranchè dans les forêts traversées $(12 \mathrm{~m}$. à $15 \mathrm{~m}$.), diminue sérıeusemenl le risque d'incendie des supports de ligne; si, de plus, dans celle tranchée, on place des supports incombustıbles, tous les risques de celle espèce disparaîtront.

De bonnes fondations, un calcul sérieux des supports d"une ligne, en tenant compte des angles, des différences de portées, de la violence des vents, de la neige, elc, rendront presque impossible le renversement des supports sous l'action des inlempéries.

Le tracé devia être étudié de façon à eviter toule action des eaux sur les fondations.

3. Isolateurs brisés. Les isolateurs pouvent être brisés par malveillance (coups de fusils, coups de pierres), par l'effel de la traction des fils, par les surtensions et par suite d'effels de dilatation. - Linumérer ces causes d'acsidents, c'est énumérer les qualités que l'on dort demander aux isolateurs. Cenx-ci doivent salisfarre, non seulement aux essass électriques yue nous indıquerons plus loin, mais aussi à des essais mécanıques sévères, ayant pour but de s'assurer que les isolateurs résisteront bien à la lraction des fils, et même dans une cerlaine mesure aux coups de fusils.

Pour éviler le bris des isolateurs par dilatation, il y a lieu d'examiner les dilatations relatives de la porcelaine de l'ısolateur et du mastic employé, tant pour le scellement des cloches entre elles, que pour le sceliement de l'isolateur complet sur sa ferrure. En plongeant l'ssolateur scellé, successivement dans l'eau chaude el dans l'eau froide, et en réalisant des différences de température de $85^{\circ}$ à $90^{\circ}$, on s'assurera que le mastic employé est bon. En général, le ciment de Portland donne de bons résultats avec tous genres de porcelaines. Le mélange de litharge et de glycérine donne de bons résultats avec certaines porcelaines, et mauvais avec d'autres; le soufre donne de mauvais résultats, ou de médiocres résultats, avec presque toutes les porcelaines.

4. Accıdents dus $\dot{a}$ la foudre. - L'électricité atmosphérique $\dot{a}$ de multiples moyens d'action sur les lignes de transport de force. Eile peut agir :

Par induclion électrostatique : par exemple, lorsqu'un nuage chargé d'électricité passe au-dessus d'une ligne;

Par induction électromagnétique : Iors de décharges entre deur nuages situés dans le voisınage de la ligne;

Par action directe lorsque la foudre tombe sur un support de la ligne;

Peut-être, par ionisation de l'air, dans le cas où la foudre tombe dans le voisinage de la ligrae, etc. 